Journal of Theoretical and Computational Chemistry

Vol. 4, No. 4 (2005) 1175-1181

(C) World Scientific Publishing Company

\title{
AUTHOR INDEX VOLUME 4 (2005)
}

Berlu, L. \& Safouhi, H., Analytical development of multicenter overlap-like quantum similarity integrals over slater type orbitals and numerical evaluation

Bowman, J.M., see Park, S.C.

Braams, B.J., see Park, S.C.

Cai, Z., see He, M.

Cao, X. \& Dolg, M., Pseudopotential studies on the electronic structure of lanthanum monohalides $\mathrm{LaF}, \mathrm{LaCl}, \mathrm{LaBr}$, and LaI

4 (2005) 787-801

4 (2005) 163-173

4 (2005) 163-173

4 (2005) 383-395

Cao, Z., Jin, X. \& Zhang, Q., Density functional study of the structure of the FeMo cofactor with an interstitial atom and homocitrate ligand ring opening

Carreira, L.A., see Whiteside, T.S.

Chaudhuri, R.K., see Das, B.P.

Chen, Z., see Wang, Z.-X.

Cho, C.H., see Kim, H.

Chuev, G.N., Fedorov, M.V., Luo, H.J., Kolb, D. \& Timoshenko, E.G., 3D wavelet treatment of solvated bipolaron and polaron

Chulkov, E., see Zuluaga, J.J.

Chung, L.W. \& Wu, Y.-D., A theoretical study on the intermolecular hydroacylation of alkyne catalyzed by cationic rhodium complex

Ciobanu, C., see Shih, A.

Dang, Z., see Lu, G.-N.

Das, B.P., Latha, K.V.P., Sahoo, B.K., Sur, C., Chaudhuri, R.K. \& Mukherjee, D., Relativistic and correlation effects in atoms

Dholabhai, P.P. \& Ray, A.K., An ab initio study of charge state effects on the use of $\mathrm{Ag}_{2}^{+}$and $\mathrm{Ag}_{2}$ for catalytic oxidation of $\mathrm{CO}$

4 (2005) 583-592

4 (2005) 593-602

4 (2005) 461-474

4 (2005) $1-20$

4 (2005) 669-688

4 (2005) 305-315

4 (2005) 751-767

4 (2005) 357-372

Ding, W.-J., Ni, L.-Y., Fang, W.-H. \& Yu, J.-G., Theoretical study on the unimolecular reactions of glyoxylic acid

Ding, Y.-H., see Xie, H.-B.

Dobrescu, G., Fangli, I. \& Rusu, M., Simulation of CO chemisorption on Pt supported on fractal surfaces

Dolg, M., see Cao, X.

Downton, M.T., see Wang, F.

Duan, Y., see Wang, Z.-X.

Eid, Kh. M., see Shalabi, A.S.

4 (2005) 737-749

4 (2005) 1101-1117

4 (2005) 811-822

4 (2005) 1-20

4 (2005) 1075-1091

4 (2005) 725-736

4 (2005) 1029-1055

4 (2005) 769-785

4 (2005) 583-592

4 (2005) 247-264

4 (2005) 689-705

4 (2005) 883-905 
Fang, G.-Y., Xu, L.-N., Hu, X.-G., Li, X.-H., Xiao, H.-M., Ju, X.-H. \& Gong, X.-D., Density functional theory study of the interaction between 3-nitro-1,2,4-triazole-5-one and water

$4(2005) 849-856$

Fang, W.-H., see Ding, W.-J.

Fangli, I., see Dobrescu, G.

Fedorov, M.V., see Chuev, G.N.

Feng, D., see He, M.

Fu, K.-X., see Li, X.-Y.

García-Granda, S., see Van der Maelen Uría, J.F.

Ghosh, D.C., A new scale of electronegativity based on absolute radii of atoms

Glaser, R., see $\mathrm{Wu}, \mathrm{Z}$.

Gong, X.-D., see Fang, G.-Y.

$\mathrm{Gu}, \mathrm{R}$., see Weber, K.H.

Han, K.-L., see Wang, D.

Harris, J.A., see Weber, K.H.

Hartke, B., see Tekin, A.

Hayashi, M., see Mishima, K.

He, M., Feng, D., Xie, J. \& Cai, Z., Computational studies of the ammonolysis for $\mathrm{N}$-methyl $\beta$-sultam

Hirao, K., see Yanagisawa, S.

Hiyama, M. \& Kosugi, N., Ab initio R-matrix/multi-channel quantum defect theory approach to study molecular core excitation and ionization: GSCF4R

4 (2005) 725-736

4 (2005) 769-785

4 (2005) 751-767

4 (2005) 383-395

4 (2005) 907-983

4 (2005) 823-832

4 (2005) 21-33

4 (2005) 373-381

4 (2005) 849-856

4 (2005) 623-638

4 (2005) 857-865

4 (2005) 623-638

4 (2005) 1119-1151

4 (2005) 1153-1163

4 (2005) 383-395

4 (2005) 265-280

$4(2005) 35-47$

Hori, T., Takahashi, H. \& Nitta, T., Hybrid quantum mechanical/molecular mechanical approach to enzymatic reactions by utilizing the real-space grid technique

$\mathrm{Hu}, \mathrm{C}$. , see Qin, S.

Hu, X.-G., see Fang, G.-Y.

Jalili, S. \& Moradi, F., Charge transport through thiophene bithiol molecule as a molecular wire

Jiao, H., see Wang, Z.-X.

Jin, X., see Cao, Z.

Ju, X.-H., see Fang, G.-Y.

Katouda, M., Kobayashi, M., Nakai, H. \& Nagase, S., Practical performance assessment of accompanying coordinate expansion recurrence relation algorithm for computation of electron repulsion integrals

Kidwani, N., see Wang, F.

Kikuchi, Y., see Nakai, H.

Kim, H., Cho, C.H. \& Lee, E.K., Dynamics of simple fluids confined in cylindrical pore: Effect of pore size

Kirk, W.R., The steady-state, ergodicity, and time-ordering in simulations on families of chemical reactions

Kita, Y., Wako, K., Okada, I. \& Tachikawa, M., Ab initio calculations of intermolecular interaction potentials of fullerene-fragments systems

Kobayashi, M., see Katouda, M.

Koga, N., see Suresh, C.H.

Kolb, D., see Chuev, G.N.

4 (2005) 867-882

4 (2005) 449-459

4 (2005) 849-856

4 (2005) 1001-1014

4 (2005) 669-688

4 (2005) 593-602

4 (2005) 849-856

4 (2005) 139-149

4 (2005) 247-264

4 (2005) 317-331

4 (2005) 305-315

4 (2005) 475-492

4 (2005) 49-58

4 (2005) 139-149

4 (2005) 59-73

4 (2005) 751-767 
Kondorskiy, A. \& Nakamura, H., Semiclassical formulation of optimal control theory

$4(2005) 75-87$

Kondorskiy, A. \& Nakamura, H., Semiclassical frozen Gaussian propagation method for electronically nonadiabatic chemical dynamics: Møller operator formulation and incorporation of the Zhu-Nakamura theory

Kosugi, N., see Hiyama, M.

Kroes, G.-J. \& Somers, M.F., Six-dimensional dynamics of dissociative chemisorption of $\mathrm{H}_{2}$ on metal surfaces

Kurita, N., see Sugiki, S.-I.

Kwak, J. \& Lee, Y.S., A computational grid system for quantum chemical calculations tested in a modeling of the $\mathrm{GE}(001)$ surface

Laio, A., see Sulpizi, M.

Larson, L.J., see Weber, K.H.

Latha, K.V.P., see Das, B.P.

Lee, E.K., see Kim, H.

Lee, Y.S., see Kwak, J.

Leiva, P. \& Piris, M., A natural orbital functional study for the electric response properties of molecules

Li, Q., see Shuai, Z.

Li, S., see Weber, K.H.

Li, X.-H., see Fang, G.-Y.

Li, X.-Y. \& Fu, K.-X., Continuous medium theory for nonequilibrium solvation: New formulations and an overview of theories and applications

Lin, S.H., see Mishima, K.

Liu, S., see Zhong, A.

Lo, C.F., see Yeung, M.Y.

Lu, G.-N., Dang, Z., Tao, X.-Q., Peng, P.-A. \& Zhang, D.-C., QSPR study on direct photolysis half-lives of PAHs in water surface

Luo, H.J., see Chuev, G.N.

Ma, N.L. \& Wu, P., A density functional study of the structure of silane coupling agent 3-aminopropyltriethoxysilane

Mahecha, J., see Zuluaga, J.J.

Matsuoka, M., see Sugiki, S.-I.

Minamino, S., see Tokue, I.

Mishima, K., Hayashi, M. \& Lin, S.H., Quantum interference and laser pulse phase effect on the photoionization rates of excited hydrogen atoms in the tunneling region

Miyoshi, E., see Mori, H.

Moradi, F., see Jalili, S.

Mori, H. \& Miyoshi, E., Possibility of molecular-switch with controlled hydrogen bond: Utility of combination of $2,2^{\prime}$-biimidazole and redox-active ligand

4 (2005) 89-102

4 (2005) 35-47

4 (2005) 493-581

4 (2005) 183-195

4 (2005) 289-303

4 (2005) 985-999

4 (2005) 623-638

4 (2005) 1-20

4 (2005) 305-315

4 (2005) 289-303

4 (2005) 1165-1173

4 (2005) 603-622

4 (2005) 623-638

4 (2005) 849-856

4 (2005) 907-983

4 (2005) 1153-1163

4 (2005) 833-847

4 (2005) 103-115

4 (2005) 811-822

4 (2005) 751-767

4 (2005) 117-126

4 (2005) 357-372

4 (2005) 183-195

4 (2005) 225-245

4 (2005) 1153-1163

4 (2005) 333-344

4 (2005) 1001-1014

4 (2005) 333-344

4 (2005) 639-655

Morokuma, K., see Witek, H.A.

Mukherjee, D., see Das, B.P.

4 (2005) $1-20$ 
Murata, K., Sugita, Y. \& Okamoto, Y., Molecular dynamics simulations of DNA dimers based on replica-exchange umbrella sampling. I. Test of sampling efficiency

Murata, K., Sugita, Y. \& Okamoto, Y., Molecular dynamics simulations of DNA dimers based on replica-exchange umbrella sampling. II. Free energy analysis

4 (2005) 433-448

Nagase, S., see Katouda, M.

Nakai, H. \& Kikuchi, Y., Extension of energy density analysis to treating chemical bonds in molecules

Nakai, H., see Katouda, M.

Nakamura, H., see Kondorskiy, A.

Nakamura, H., see Kondorskiy, A.

Nakamura, H., Nonadiabatic transition and chemical dynamics: Multi-dimensional tunneling theory and applications of the Zhu-Nakamura theory

Nanbu, S., see Tokue, I.

Ni, L.-Y., see Ding, W.-J.

Nitta, T., see Hori, T.

Ojha, D.P., Ordering in higher homologous series of $n \mathrm{BAC}-\mathrm{A}$ comparative computational analysis

Ojha, D.P., Ordering of a thermotropic mesogen at phase transition temperature - a statistical approach based on quantum mechanics

Okada, I., see Kita, Y.

Okamoto, Y., see Murata, K.

Okamoto, Y., see Murata, K.

Parasuk, V. \& Unarunotai, S., Solvent effects of kinetics of $[1,5]$ $\mathrm{H}$-shift in cyclopentadiene and its derivatives

Park, S.C., Braams, B.J. \& Bowman, J.M., Construction of a global potential energy surface from novel $a b$ initio molecular dynamics for the $\mathrm{O}\left({ }^{3} \mathrm{P}\right)+\mathrm{C}_{3} \mathrm{H}_{3}$ reaction

Peng, P.-A., see Lu, G.-N.

Pesonen, J., Hamiltonians in oblique body-frame: A geometric algebra approach

Piris, M., see Leiva, P.

Qin, S., Hu, C. \& Yang, H., Theoretical study on the mechanism of the reaction of $\mathrm{Ni}\left(\mathrm{d}^{101} \mathrm{~S}\right)+\mathrm{H}_{2}+\mathrm{CO}_{2} \rightarrow \mathrm{NiCO}+\mathrm{H}_{2} \mathrm{O}$

Ray, A.K., see Dholabhai, P.P.

Richardson, N.A., see Temelso, B.

Rothlisberger, U., see Sulpizi, M.

Ruiz, J., see Van der Maelen Uría, J.F.

Rusu, M., see Dobrescu, G.

Safouhi, H., see Berlu, L.

Sahoo, B.K., see Das, B.P.

Sari, L., see Temelso, B.

Scemama, A., Investigating the volume maximizing the probability of finding $\nu$ electrons from variational Monte Carlo data

Schaefer III, H.F., see Temelso, B.

Schleyer, P.V.R., see Wang, Z.-X.

4 (2005) 317-331

4 (2005) 139-149

4 (2005) $75-87$

4 (2005) 89-102

4 (2005) 127-137

4 (2005) 225-245

4 (2005) 725-736

4 (2005) 867-882

4 (2005) 1015-1028

4 (2005) 803-810

4 (2005) 49-58

4 (2005) 411-432

4 (2005) 433-448

4 (2005) 151-161

4 (2005) 163-173

4 (2005) 811-822

4 (2005) 1057-1074

4 (2005) 1165-1173

4 (2005) 449-459

4 (2005) 1075-1091

4 (2005) 707-724

4 (2005) 985-999

4 (2005) 823-832

4 (2005) 769-785

4 (2005) 787-801

4 (2005) 1-20

4 (2005) 707-724

4 (2005) 397-409

4 (2005) 707-724

4 (2005) 669-688 
Schwartz, S.D., A new semiclassical dynamics from the interaction representation

Sekino, H., see Sugiki, S.-I.

Sengoku, Y., see Sugiki, S.-I.

Shalabi, A.S. \& Eid, Kh. M., The role of oxidation states in laser light generation and color image formation (II) an $a b$ initio study of $F_{\mathrm{A}}: \mathrm{Hg}^{+}$and $F_{\mathrm{A}}: \mathrm{Hg}^{++}$color centers at the low coordination (100) and (110) surfaces of AgBr crystal

Shiga, A., see Yamanaka, M.

Shiga, M., see Tachikawa, M.

Shiga, M., see Takayanagi, T.

Shih, A., Ciobanu, C. \& Tao, F.-M., Theoretical mechanisms and kinetics for the reaction of dimethyl sulfide and ozone in water vapor

Shuai, Z., Li, Q. \& Yi, Y., Coupled-cluster equation of motion study for the electronic and optical properties of conjugated systems

So, S.K., see Yeung, M.Y.

Somers, M.F., see Kroes, G.-J.

Stradomska, A., see Witek, H.A.

Sugiki, S.-I., Matsuoka, M., Usuki, R., Sengoku, Y., Kurita, N., Sekino, H. \& Tanaka, S., Density functional calculations on the interaction between catabolite activator protein and cyclic AMP using the fragment molecular orbital method

4 (2005) 1093-1100

4 (2005) 183-195

4 (2005) 183-195

4 (2005) 883-905

4 (2005) 345-355

4 (2005) $175-181$

4 (2005) 197-207

4 (2005) 1101-1117

4 (2005) 603-622

4 (2005) 103-115

4 (2005) 493-581

4 (2005) 639-655

Sugita, Y., see Murata, K.

Sugita, Y., see Murata, K.

Sulpizi, M., Rothlisberger, U. \& Laio, A., Electron transfer induced dissociation of chloro-cyano-benzene radical anion: Driving chemical reactions via charge restraints

Sun, C.-C., see Xie, H.-B.

Sur, C., see Das, B.P.

Suresh, C.H. \& Koga, N., Oxidative addition of $\mathrm{C}-\mathrm{X}$ bond in $\mathrm{HCCX}\left(\mathrm{X}=\mathrm{H}, \mathrm{CH}_{3}\right.$, and $\left.\mathrm{SiH}_{3}\right)$ to Rhodium(I) complex and subsequent 1,3-migration of X: A DFT level study

Tachikawa, M. \& Shiga, M., Ab initio path integral study on isotope effect of ammonia molecule

Tachikawa, M., see Kita, Y.

Takahashi, H., see Hori, T.

Takayanagi, T., Shiga, M. \& Taketsugu, T., Development of a three-dimensional $a b$ initio potential energy surface for the $\mathrm{He}-\mathrm{Cl}_{2}(\mathrm{X})$ system and its application to solvation structures in the $\mathrm{He}_{n} \mathrm{Cl}_{2}$ clusters

Taketsugu, T., see Takayanagi, T.

Tanaka, S., see Sugiki, S.-I.

Taniike, T. \& Yamashita, K., A flux-flux cross-correlation function approach to self-diffusion of hydrogen atom on a CU(111) surface

4 (2005) 183-195

4 (2005) 411-432

4 (2005) 433-448

4 (2005) 985-999

4 (2005) 1029-1055

4 (2005) $1-20$

4 (2005) 59-73

4 (2005) 175-181

4 (2005) 49-58

4 (2005) 867-882

4 (2005) 197-207

4 (2005) 197-207

4 (2005) 183-195

Tao, F.-M., see Shih, A.

Tao, F.-M., see Weber, K.H.

Tao, X.-Q., see Lu, G.-N.

4 (2005) 209-223

4 (2005) 1101-1117

4 (2005) 623-638

4 (2005) 811-822 
Tekin, A. \& Hartke, B., Global geometry optimization of silicon clusters employing empirical potentials, density functionals, and $a b$ initio calculations

Temelso, B., Richardson, N.A., Sari, L., Yamaguchi, Y. \& Schaefer III, H.F., Characterization of the $\tilde{X}^{2} \Pi$ and $\tilde{A}^{2} \Sigma^{+}$ electronic states of the phosphaethyne cation $\left(\mathrm{HCP}^{+}\right)$

Timoshenko, E.G., see Chuev, G.N.

Tokue, I., Yamasaki, K., Minamino, S. \& Nanbu, S., Theoretical transition probabilities for the $\tilde{A}^{2} A_{1}-\tilde{X}^{2} B_{1}$ system of $\mathrm{H}_{2} \mathrm{O}^{+}$and $\mathrm{D}_{2} \mathrm{O}^{+}$and related Franck-Condon factors based on global potential energy surfaces

Truong, T.N., see Wang, Y.

Tsuneda, T., see Yanagisawa, S.

Unarunotai, S., see Parasuk, V.

Usuki, R., see Sugiki, S.-I.

Van der Maelen Uría, J.F., Ruiz, J. \& García-Granda, S., Theoretical characterization of a highly electrophilic carbene

Wako, K., see Kita, Y.

Wang, D., Yang, M., Han, K.-L. \& Zhang, D., The failure of CS approximation in quantum reaction scattering with double deep well: Time-dependent calculation for $\mathrm{O}+\mathrm{NH}$ reaction

Wang, F., Downton, M.T. \& Kidwani, N., Adenine tautomer electronic structural signatures studied using dual space analysis

Wang, Y. \& Truong, T.N., Correlation between electronic structures of metal-intercalated single wall carbon nanotubes with their field emission properties

4 (2005) 707-724

4 (2005) 751-767

4 (2005) 225-245

4 (2005) 657-668

4 (2005) 265-280

4 (2005) 151-161

4 (2005) 183-195

4 (2005) 823-832

4 (2005) 49-58

4 (2005) 857-865

4 (2005) 247-264

4 (2005) 657-668

Wang, Z.-X. \& Duan, Y., Direct interaction energy: A computational quantity for parameterization of condensed-phase force fields and its application to hydrogen bonding

Wang, Z.-X., Chen, Z., Jiao, H. \& Schleyer, P.V.R., Isolobal boron carbonyl carbocation analogs

Weber, K.H., Harris, J.A., Larson, L.J., Tao, F.-M., Li, S. \& $\mathrm{Gu}, \mathrm{R}$., Mechanistic study of the reactions of sulfur trioxide with hydrogen halides to form superacid and survey of halo-sulfonic acid strength

Whiteside, T.S. \& Carreira, L.A., Prediction of the enthalpy of formation of halogenated hydrocarbons using SPARC

Witek, H.A., Morokuma, K. \& Stradomska, A., Modeling vibrational spectra using the self-consistent charge density-functional tight-binding method II. Infrared spectra

Wu, P., see Ma, N.L.

Wu, Y.-D., see Chung, L.W.

$\mathrm{Wu}, \mathrm{Z}$. \& Glaser, R., Additivity schemes in conformational analysis. Concept and demonstration

Xiao, H.-M., see Fang, G.-Y.

Xie, H.-B., Ding, Y.-H. \& Sun, C.-C., Radical-molecule reactions $\mathrm{HCO} / \mathrm{HOC}+\mathrm{C}_{2} \mathrm{H}_{4}$ : A mechanistic study

4 (2005) 689-705

4 (2005) 669-688

4 (2005) 623-638

4 (2005) 461-474

Xie, J., see He, M.

4 (2005) 639-655

4 (2005) $117-126$

4 (2005) 737-749

4 (2005) 373-381

4 (2005) 849-856

4 (2005) 1029-1055

4 (2005) 383-395 
Xu, L.-N., see Fang, G.-Y.

4 (2005) 849-856

Yamaguchi, Y., see Temelso, B.

4 (2005) 707-724

Yamanaka, M. \& Shiga, A., A new orbital interaction analysis system "LUMMOX" and it's application of phosphine-palladium complexes

4 (2005) 345-355

Yamasaki, K., see Tokue, I.

4 (2005) 225-245

Yamashita, K., see Taniike, T.

Yanagisawa, S., Tsuneda, T. \& Hirao, K., Investigation of

4 (2005) 209-223 dominant electron configurations in time-dependent density functional theory

Yang, H., see Qin, S.

Yang, M., see Wang, D.

Yang, Z.-Z., see Zhang, M.-B.

Yeung, M.Y., Lo, C.F. \& So, S.K., An ab initio study of carbazole model compounds

Yi, Y., see Shuai, Z.

Yu, J.-G., see Ding, W.-J.

Zhang, D., see Wang, D.

Zhang, D.-C., see Lu, G.-N.

Zhang, M.-B., Zhao, D.-X. \& Yang, Z.-Z., The characteristic boundary radii of atoms

Zhang, Q., see Cao, Z.

Zhao, D.-X., see Zhang, M.-B.

Zhong, A. \& Liu, S., Validity and interpretation of hund's multiplicity rule for molecules: A density functional study

Zuluaga, J.J., Mahecha, J. \& Chulkov, E., Semi-classical propagation and spectral analysis in the $\mathrm{H}^{-}$ion interacting with a metallic surface

4 (2005) 265-280

4 (2005) 449-459

4 (2005) 857-865

4 (2005) 281-288

4 (2005) 103-115

4 (2005) 603-622

4 (2005) 725-736

4 (2005) 857-865

4 (2005) 811-822

4 (2005) 281-288

4 (2005) 593-602

4 (2005) 281-288

4 (2005) 833-847

4 (2005) 357-372 